\title{
CyPE is an essential element of allergen cyclophilin family
}

\author{
Yuwei $\mathrm{Li}^{1}$, Yuwei Wang ${ }^{1}$, Peixin $\operatorname{Ran}^{2}$, and ZhiGang Liu ${ }^{3}$ \\ ${ }^{1}$ Shenzhen University \\ ${ }^{2}$ guangzhou medical college \\ ${ }^{3}$ State Key Laboratory of Respiratory Disease for Allergy at Shenzhen University Shenzhen
}

September 11, 2020

\begin{abstract}
Pan-allergens can contribute to severity and perpetuation of allergic diseases, as a result of leading to IgE-medicated polysensitization and cross-reactivity to human self-antigens. Cyclophilins are well-known pan-allergens of various groups of crossreactive allergens. CyPs have been identified with five classic CyP isoforms (CyPA, B, C, D and E). In the present study, the physicochemical and immunologic characteristics of CyPs were predict by bioinformatics and immunoinformatics approaches. The results indicated that CyPA family possesses multiple identified allergens. CyPE showed closer evolutionary relationship and high sequence identity with allergenic CyPA and the structure and antigenicity of CyPE were conserved significantly compared with allergenic CyPA. To verify results of In silico analysis, CyPE from HMD were successfully constructed and purified. ELISA resuls indicated that 2/14 patient serum had positive reactions to CyPE. CyPE is low antigenicity but sufficient to induced significant airway inflammation in mouse models and IgE, IgG1, eosinophil infiltration, IL-4 and co-stimulatory molecules of CyPE were markedly lower levels than Der f 29. Structure and immunoinformatics analysis demonstrated that RNA-binding motif of CyPE may reduce the immunogenicity of $\mathrm{CyPE}$ and structure variation of B-cell epitopes is the reason why CyPB is no IgE activity. This work is of significance in the diagnosis and treatment of allergy.
\end{abstract}

$\mathrm{CyPE}$ is an essential element of allergen cyclophilin family

Yuwei $\mathrm{Li}^{1,2,3}$, Yuwei Wang ${ }^{2}$, Peixin $\operatorname{Ran}^{3^{*}}$, and Zhigang $\mathrm{Liu}^{1,2^{*}}$

${ }^{1}$ Department of Respirology \& Allergy. The Third Affiliated Hospital of Shenzhen University, Shenzhen 518020, China.

${ }^{2}$ State Key Laboratory of Respiratory Disease for Allergy at Shenzhen University, Shenzhen University School of Medicine, Shenzhen 518060, China.

${ }^{3}$ State Key Laboratory of Respiratory Disease, Guangzhou Medical College, Guangzhou, 510006.

* Correspondence: Correspondence and requests for materials should be addressed to Z.L. (email: lzg@szu.edu.cn).

Abstract

Pan-allergens can contribute to severity and perpetuation of allergic diseases, as a result of leading to IgE-medicated poly-sensitization and cross-reactivity to human self-antigens. Cyclophilins are well-known pan-allergens of various groups of cross-reactive allergens. CyPs have been identified with five classic CyP isoforms (CyPA, B, C, D and E). In the present study, the physicochemical and immunologic characteristics of $\mathrm{CyPs}$ were predict by bioinformatics and immunoinformatics approaches. The results indicated that CyPA family possesses multiple identified allergens. CyPE showed closer evolutionary relationship and high sequence identity with allergenic CyPA and the structure and antigenicity of CyPE were conserved significantly compared with allergenic CyPA. To verify results of In silico analysis, CyPE from HMD were 
successfully constructed and purified. ELISA resuls indicated that 2/14 patient serum had positive reactions to CyPE. CyPE is low antigenicity but sufficient to induced significant airway inflammation in mouse models and IgE, IgG1, eosinophil infiltration, IL-4 and co-stimulatory molecules of CyPE were markedly lower levels than Der f 29. Structure and immunoinformatics analysis demonstrated that RNA-binding motif of CyPE may reduce the immunogenicity of $\mathrm{CyPE}$ and structure variation of $\mathrm{B}$-cell epitopes is the reason why $\mathrm{CyPB}$ is no $\operatorname{IgE}$ activity. This work is of significance in the diagnosis and treatment of allergy.

Keywords: Cyclophilin; CyPE; Allergen; Epitope; Antigenicity

\section{Introduction}

Pan-allergens were various groups of cross-reactive allergens and represent proteins sharing a high degree of sequence and structural homology, playing very important roles in IgE-mediated poly-sensitization ${ }^{1}$. Panallergens often show IgE-medicated cross-reactivity to human self-antigens, which can contribute to severity and perpetuation of allergic diseases ${ }^{2}$. Cyclophilins $(\mathrm{CyPs})$ are well-known pan-allergens and possess wide ranging cross-reactivity ${ }^{3}$. Furthermore, CyPs is the major intracellular receptor for cyclosporin A (CsA) ${ }^{4-5}$, which is one of the most important immunosuppressant drugs used for prevention of graft rejection after transplant surgery ${ }^{5-6}$. Binding of the CsA-Cyp complex to calcineurin inhibits the phosphatase activity of calcineurin $^{7}$, preventing the translocation of nuclear factor of activated T cells (NF-AT) from the cytosol to the nucleus leading to attenuat the $\mathrm{T}$ cell-mediated graft rejection ${ }^{8-9}$.

CyPs possess peptidyl-prolyl cis-trans-isomerase (PPIase) activity (PPIase, EC 5.2.1.8) ${ }^{10}$. CyPs have been identified with size ranging from 18 to $40 \mathrm{kDa}$ and among which, the functions of five classic CyP isoforms (CyPA, B, C, D and 33) are mostly studied ${ }^{4,11}$. CyPA of HDM was reported as a main allergen,Cyclophilins are found in all cells of all organisms studied includeing mammals, plants, insects, fungi, and bacteria ${ }^{12}$. Pci c 2 (from Psilocybe cubensis), Asp f 11 (from Aspergillus fumigatus), Mal f 6 (from Malassezia furfur), Bet $\mathrm{v} 7$ (from birch), and Cat $\mathrm{r} 1$ (from Catharanthus roseus) have been recognized as an IgE-binding protein 13-14. While CyPB demonstrates no IgE activity. Immune response and characterization of structure and epitopes of HDM CyP will be beneficial for the diagnosis and treatment of mite-induced atopic illnesses and the effective epitope-based vaccine design.

In the present study, To clarify the feature and diversity of CyPs family allergens,we analyzed the sequence, structures and immunologic characteristics of different source CyPs by using bioinformatics methods. The allergenicity of $\mathrm{CyPs}$ of HDM was identified with a mouse model and in vitro cell experiments.

\section{Materials and Methods}

\subsection{Materials}

The using antibodies for measuring IgE and IgG1 were purchased from Southern biotech, USA. PE-CD80, PE-CD83 and MHC II antibodies were purchased from Ebioscience, USA (12-0801, 12-0831 and 11-5321). Aluminum hydroxide and Thetetramethylbenzidine (TMB) was purchased from Thermo Fisher, USA (77161) and Solarbio, China (R1200), respectively. ELISA kits for IL-4 and IFN- $\gamma$ detection were purchased from Ebioscience, USA (88-7044 and 88-7314). PerCP-CD4, FITC-IL-4, PE-IFN- $\gamma$, PE-CD11c and PerCP-SiglecF were obtained from Ebioscience, USA (46-0041, 11-7042, 12-7311, 12-0114 and 46-1702). All other chemicals were all purchased from Sigma-Aldrich (St. Louis, MO, USA). All other chemicals were of analytical grade unless otherwise stated.

\subsection{Phylogenetic analysis and Homology Modeling}

The homologous amino acid sequences were selected by BLAST in NCBI and Uniprot. Phylogenetic tree was generated by using Neighbor Joining (NJ) phylogenetic analysis on the basis of the JTT amino acid sequence 
distance implemented in MEGA $5.1^{15}$. The multiple alignment of homologous amino acid sequences was performed using Clustal X 2.1 program ${ }^{16}$. Physicochemical analysis of the CyPs protein sequences was predicted by ProtParam ${ }^{17}$. Secondary structural of CyPs were predicted by PSIPRED ${ }^{18}$. The protein sequence of CyPs was used to search for homology in the PDB. The suitable templates for CyPs were selected by SWISS-MODEL server (http://swissmodel.expasy.org/ $)^{19}$. The protein structure of CyPs was built through alignment mode in SWISS-MODEL by using the best template. An initial structural model was checked for recognition of errors in 3D structure by PROCHECK , ERRAT , and VERIFY 3D programs ${ }^{20}$. The final model structure quality of CyPs was assessed by QMEAN, Ramachandran plots, and ProSA program ${ }^{21}$.

\subsection{In Silico Prediction of Epitopes.}

Cbtope, BCPred, DNAStar protean system and BepiPred 1.0 server were used to predict B-cell epitopes of CyPs. The consensus epitopes were accurate through combining the results of the four tools, as the ultimate predicting results of B-cell epitopes with a previously published method ${ }^{22}$. Cbtope predicted B-cell epitopes in antigen sequences, using an artificial neural network ${ }^{23}$. BCPred, DNAStar protean system and BepiPred 1.0 server only need the amino acid sequence and provide more straightforward results ${ }^{24}$.

\subsection{Cloning, expression, purification of HDM CyPs}

For cloning and expression of the CyPs gene,E. coli DH5 (Invitrogen, Carlsbad, CA, USA) and E. coli Rosetta-gami (DE3) (Novagen, Madison, WI, USA) were used as hosts of vector. The plasmid pET32a $(+)$ (Novagen, Madison, WI, USA) was used as the CyPs gene vector. The gene of CyPs was obtained from the whole genome of $H D M$. The cDNA product of $\mathrm{CyPs}$ was obtained by reverse transcription kits and subcloned into pET-32a(+) and expressed in E. coli Rosetta-gami (DE3) cells. Expression of CyPs was induced by adding $0.1 \mathrm{mM}$ isopropyl- $\beta$-D-thiogalactopyranoside (IPTG) at 30 for $16 \mathrm{~h}$, when the cells were an absorbance of 0.8 at $\mathrm{OD} 600 \mathrm{~nm}$. The recombinant $\mathrm{CyPs}$ was purified by affinity chromoatography. An ion exchange column and ToxinEraser Endotoxin Removal Kit (L00338, Genscript, China) was used to remove the endotoxin. The LPS concentration was lower than $0.1 \mathrm{EU} / \mathrm{ml}$ after detected by the Chromogenic LAL Endotoxin Assay Kit (L00350C, Genscript, China). The recombinant protein was analyzed by $12 \%$ SDS-PAGE stained with Coomassie Blue. The recombinant proteins were collected and stored at $4^{\circ} \mathrm{C}$.

\subsection{ELISA for analysis of IgE reactivity}

The serum of patients with dust-mite allergic disease were obtained from the First Affiliated Hospital of Guangzhou Medical University. The serum from non-allergic persons were used as controls. Each serum sample was provided a written informed consent from the participant. The Human Ethic Committee at Shenzhen University (Shenzhen, Guangdong, China) approved the using human sera. All methods were carried out in accordance with relevant guidelines and regulations or Declaration Helsinki in the present study. The antigen-specific IgE in patient serum to CyPs was detected by indirect ELISA. The 96 well plates were coated with $100 \mu \mathrm{L}$ of mixture sample for $12 \mathrm{~h}$ at 4 . Then, $200 \mu \mathrm{L}$ of $3 \%$ BSA in PBS was used for blocking the plate at 37 for $2 \mathrm{~h}$. The allergic serum to dust mite (diluted to five times) was incubated overnight at 4 . The plates were incubated at 37 with a peroxidase-labeled mouse anti-human IgE $(1: 2,000)$ monoclonal antibody for $60 \mathrm{~min}$ and were then washing six times with PBST. The plates were subjected to color development and terminated by $2 \mathrm{M} \mathrm{H}_{2} \mathrm{SO}_{4}$. The absorbance at $450 \mathrm{~nm}$ were measured on a microplate reader. All of the data are expressed as mean \pm SEM and processed with Graphpad software

\subsection{Mouse model of allergic asthma}

BALB/c female mice (6-8 weeks, 18-20g) were purchased from the Guangdong Experimental Animal Center (Guangzhou, Guangdong, China), and the mice were maintained in a pathogen-free facility at Shenzhen University. Experimental procedures were approved by the Animal Ethics Committee at Shenzhen University. All methods were carried out in accordance with relevant guidelines and regulations for using mouse in the study. 24 mice were divided into four groups: CyPA group, CyPE group, Control group. The mice were subcutaneously immunized with $50 \mu \mathrm{g}$ CyPs mixing with 0.3 mlaluminum hydroxide, or PBS on 0 , 3rd 
and 7th day, respectively. CyPs group mice were challenged with CyPs protein via intranasal instillation next week, respectively. On day 23 , the mice were sacrificed. The excised lung tissue was fixed in $4 \%$ paraformaldehyde. The paraffin was used to embed the dehydrated lung tissue. The lung tissue of $5 \mu \mathrm{m}$ thick sections were cut and fixed on glass slides. The lung tissue specimen was observed by light microscope after stained with Hematoxylin and Eosin (HE). Eosinophils in Bronchoalveolar lavage fluid (BALF) were assessed and counted by a flow cytometer (FACS). The levels of specific IgE and IgG1 in mouse serum and cytokines in BALF were detected by ELISA.

\subsection{DC2.4 co -stimulatory molecule and splenocytes cytokine detection.}

Dulbecco's Modified Eagle's Medium (DMEM) was used to culture DC2.4 cells with $10 \mathrm{mM}$ 4-(2hydroxyethyl)-1-piperazineethanesulfonic acid (HEPES) and 10\% fetal bovine serum (FBS) at 37 in $5 \%$ $\mathrm{CO}_{2}$. 96 well plates was used to culture DC2.4 cells $\left(2 \times 10^{5}\right.$ cells/well $)$ for $20 \mathrm{~h}$ and then add CyPs or culture medium alone for $24 \mathrm{~h}$ before assaying cytokine. We collected cells and stained with corresponding antibodies against CD80, CD83 and MHC II (Ebioscience), then assessed by a flow cytometer (FACS). Spleen cells were collected and incubated $\left(5 \times 10^{6}\right.$ cells per well) with culture medium containing $0.2 \mathrm{mg} / \mathrm{ml} \mathrm{CyPs}$ $(0.2 \mathrm{mg} / \mathrm{ml})$ or PBS for $72 \mathrm{~h}$. The levels of IL-4 and IFN- $\gamma$ in culture medium of splenocytes were assessed by FACS.

\subsection{Data statistics}

The data (mean \pm SEM) were processed with GraphPad software. The T-test was used for the mean significant differences between two groups. ${ }^{*}, \mathrm{P}<0.05$. ${ }^{* *}, \mathrm{P}<0.01$. ${ }^{* * *}, \mathrm{P}<0.001$. ns, no significant difference.

\section{Result}

\section{Bioinformatics analysis of CyPs}

Cyclophilin is a common allergen and CyPs family possesses multiple members. The feature and diversity of CyPs family allergens is not reported. Thirty-three sequences of CyPs family were obtained from the database of NCBI and UniRef to construct the evolutionary tree (Fig. 1A). The identified allergens of CyPs family are almost CyPA, such as Der f 29, Asp f 27, Asp f 11, Mala s 6, Rhi o 2, Bet v 7, Cat r 1, Ole e 15, and Sola 1 15, highlighted by red background in the evolutionary tree (Fig. 1A). Immuno-positive rate, molecular weight, amino acid composition, theoretical pIs, instability indexs, and GRAVY of CyPs family was summarize and analysised on website of ProtParam or Allergen Nomenclature. CyPE of HDM (GenBank ID: XP_027199043.1) consists of one CyP-A domain and one RNA-binding motif domain. CyP-A domain of $\mathrm{CyPE}$ encodes 165 amino acid residues with a predicted molecular weight of $18.4 \mathrm{kDa}$ and a theoretical isoelectric point (PI) of 9.35. ProtParam analysis results showed that CyP-A domain of CyPE had similar physicochemical characteristics with allergenic CyPA. Theoretical pIs, instability indexs, and GRAVY of which are $7.75-9.35,11.58-23.57$, and -0.455 to -0.07 , respectively (Table 1). Phylogenetic analysis illustrated that $\mathrm{CyPE}$ had closer evolutionary relationship with CyPA highlighted by green background in the Fig. 1A. These results illustrate that CyPE of HDM maybe a novel allergen.

\section{Allergenicity analysis of CyPs}

The three-dimensional structures of allergens promotes greatly our comprehension of allergenicitic mechanism. The three-dimensional structure of $\mathrm{CyPE}$ and CyPA of HDM was constructed through template (Fig. 1B and 1C). PROCHECK analysis, ERRAT program, VERIFY 3D program, ProSa analysis, and QMEAN analysis were used to assess model structure quality (Table 2). CyPs contained PPIase domain, which eightstranded antiparallel $\beta$-sheet surround the surface of barrel and two $\alpha$-helices clog the top and the bottom of barrel (Figure 1B and 1C). CyPE of HDM consists of two domains (one RNA-binding motif and one CyP-A 
domain) which was linked by a flexible 69 -amino-acid-long LR. The overall folding of RNA-binding motif (RBM) domain is a classical $\alpha-\beta$ sandwich structure, a $\beta-\alpha-\beta-\beta-\alpha-\beta$ topology fold, which demonstrated by the first structure (Fig. 1C). The inner core of RBM domain is a spacially arranged four-stranded antiparallel $\beta$-sheet and two $\alpha$-helices wraping $\beta$-sheet. The three-dimensional structure is contribution for analyzing allergenicity mechanism of CyPs.

In silico prediction of B-cell epitopes generally based on amino acid features of hydrophobicity, segmental flexibility, accessibility, polarity, antigenicity, exposed surface, and turns. In this paper, DNAStar, BepiPred, and Bcepred system were used to predict B-cell epitopes based on these sequence properties of CyPs (Table 3 ). The final B-cell epitopes of CyPs were shown in the Table 4 and were labeled in sequence comparison of $\mathrm{CyPE}$ and other $\mathrm{CyPs}$ allergens (Fig. 2A). The final B-cell epitopes of CyPs included four sequences except for CyPE only possess three sequences. BcePred's antigenicity analysis showed that CyPE is low antigenicity at RBM-end (Fig. 2B). RBM of CyPE may reduce the immunogenicity of CyPE. The common B-cell epitopes of CyPs were also shown in the red boxes of Fig. 2A, naming as B1, B2, and B3, which are conserved among the four CyPs. The structural $\alpha$-carbon backbones of CyPE were superimposed with allergenic CyPA. The common epitopes labeled the superimposed $\alpha$-carbon backbones structure and tertiary structure of the CyPs (Fig. 2C). These results illustrate that CyPE maybe a novel allergen.

\section{Allergenic analysis of HDM CyPs}

To better understand allergenicity mechanism of CyPs, CyPB and CyPE of HDM was cloned, expressed and purified. The gene of CyPs was obtained from the genome of HDM. The puritied CyPs was examined by SDS-PAGE (Fig. 1A). ELISA was performed using serum from 26 dust-mite allergic patients. ELISA resuls indicated that 3/14, 2/14 and 0/14 patient serum had positive reactions to Der f 29, CyPE and CyPB, respectively. The ELISA values of Der f 29 were more twice than the value CyPE. No patient serum had positive reactions to CyPB. A animal model of allergic airway inflammation was established (Fig. 4A). The serum specific sIgE and IgG1 was measured after mouse were sacrificed. A significant difference was found between the CyPE and Der f 29 group compareing to the PBS group. Der f 29 group showed higher than the CyPE group (Fig. 4B and 4C). The total cells and eosinophils in BALF counted through flow cytometry. The number of eosinophils in Der $\mathrm{f} 29$ and CyPE groups was significantly higher than control group and the eosinophils of Der f 29 group were even more plentiful than CyPE group (Fig. 4D). Lung tissue pathology was demonstrated that the damage of bronchial mucosa and plenty of exudate was clearly observed and inflammatory cell infiltration was profound around the bronchial submucosa in Der f 29 and CyPE group, while the bronchial tube of lung tissue of control group has a clear contour and is hardly infiltrated by inflammatory (Fig. 4E). The lymphocyte infiltration in the lung tissue specimens was assessed by double blind under the microscope. The scores of control, and CyPE, Der f 29 was 14, 21, and 46, respectively. These results illustrate that $\mathrm{CyPE}$ is sufficient to induced significant airway inflammation.

\section{CyPs modulates the functions of DCs and facilitates Th2 immune response}

To clarify the mechanism of different CyPs causing airway inflammation, DC phenotypes and differentiation of T-cell were characterized under stimulation of Der f 29, CyPE and PBS. Using flow cytometry, we found that stimulation of Der f 29 and CyPE was higher levels of IL-4 on the spleen cell than PBS group (Fig. $5 \mathrm{~A})$. The IFN- $\gamma$ levels of the spleen cell incubated with different $\mathrm{CyPs}$ were no significant difference from the PBS group. The results also showed that stimulation of Der f 29 and CyPE was higher levels of CD80, CD83 and MHC II on DCs than PBS group and levels of Der f 29 was slightly higher than the CyPE group

(Fig. 5B), indicating that Der f 29 and CyPE could modulate the function of DCs but the modulation of $\mathrm{CyPE}$ is weaker than the Der f 29 group.

\section{Discussion}

Cyclophilins (CyPs) are highly conserved proteins among phylogenetically distant species and involved in a wide variety of physiological and pathophysiological processes like signal transduction, cell differentiation, 
apoptosis as well as viral, bacterial and parasitic infection ${ }^{25-26}$. Cyclophilins (CyPs) are well-known panallergens and the potential to induce complex cross-reactive among species and lead to higher threaten in allergy suffers. House dust mites are important indoor allergens, eliciting allergic diseases such as asthma, rhinitis, and atopic dermatitis and other allergic diseases ${ }^{27}$. Immune response and characterization of structure and epitopes of HDM CyP will be beneficial for the diagnosis and treatment of mite-induced atopic illnesses and the effective epitope-based vaccine design.

CyPs have been identified with five classic CyP isoforms (CyPA, B, C, D and E). CyPA of HDM was reported as a main allergen Der $\mathrm{f} 29^{28}$, while CyPB demonstrates no IgE activity ${ }^{29}$. In order to elucidate the allergic mechanism of $\mathrm{CyP}$, the physicochemical and immunologic characteristics of several classic $\mathrm{CyP}$ were analyzed through bioinformatics approaches and allergenic databases. The results showed that CyPE was closer evolutionary relationship and high sequence identity with allergenic CyPA (Fig. 1A). PPlase domain of $\mathrm{CyPE}$ were superimposed with allergenic $\mathrm{CyPA}$ in structural $\alpha$-carbon backbones (Fig. 2C). Allergens were not recognized as a whole by B- and T-cells but epitopes known as antigens. Epitopes of allergens are recognized by specific receptors present in the cell surface of B- and T-cells, leading to activate B- and T-cells ${ }^{30}$. In this paper, DNAStar, BepiPred, and Bcepred system were used to predict B-cell epitopes based on these sequence properties of Der f 29 and CyPE (Table 3). The predicted B-cell epitopes of CyPE PPlase domain demonstrated the sharing of common epitopes with the B-cell epitopes of allergenic CyPA. The structure and antigenicity analysis showed that PPlase domain of CyPE was conserved antigenic surfaces and solvent-accessibility of their putative epitopes compared with allergenic CyPA (Fig. 2).

In order to verify in silico results, CyPE from HMD were successfully constructed and purified. ELISA resuls indicated that 2/14 patient serum had positive reactions to CyPE. In order to better elucidate the mechanism of CyPs causing airway inflammation, a mouse model of allergic airways disease is characterized comprehensively. The CyPE could induce airway hyperreactivity (AHR) and markedly increased serum specific IgE and IgG1. Type 2 immune response polarization is the dominant mechanism in allergy ${ }^{31-32}$. DCs are necessary and sufficient for inducing adaptive Th2 cell responses to HDM allergen ${ }^{33-34}$. Th0 cell differentiation requires not only MHC II molecules but also co-stimulatory molecules (such as CD80, CD40 and CD86) ${ }^{35}$. In this article, the levels of CD80, CD83, and MHC II were raised significantly when DCs was stimulated with CyPE,demonstrating that CyPE could modulate the function of DCs (Fig. 5B). IL-4 levels of spleen cell were significantly higher and IFN- $\gamma$ levels were no significant difference (Fig. 5A). The levels of IFN-r and IL-4 is an important mark of Th1/Th2 imbalance ${ }^{32,36}$. These results illustrate that CyPE is low antigenicity but sufficient to induced significant airway inflammation in mouse models.

Structure analysis results showed that CyPE of HDM contained a RBM and a PPIase domain which separated by a flexible 69-amino-acid-long LR (Fig. 1C). BcePred's antigenicity analysis showed that CyPE is low antigenicity at RBM-end (Fig. 2B). RBM of CyPE may reduce the immunogenicity of CyPE. The antigenicities of $\mathrm{CyPE}$ were conserved antigenic surfaces and solvent-accessibility of their putative epitopes compared with allergenic CyPs. In addition, CyPB contain one domains CyP-B domain. The overall structure of CypB resembles that of CypA. The typical structure of cyclophilins consists of an eight-stranded antiparallel $\beta$-barrel and two $\alpha$-helices covering the top and bottom of the barrel. We inspect that CyPB was farther evolutionary relationship with allergenic CyPA from evolutionary tree (Fig. $1 \mathrm{~A}$ ). The whole structure of $\mathrm{CyPB}$ is similar to CyPA, while inserting peptides of loop1, loop2, C-end, and N-end (Fig. 6A). The main difference between CyPB and CyPA have been reported in the two loop regions (residues 19-24 and 152-164) and at the amino and carboxyl termini ${ }^{37}$. We recognize that CyPB is significant differences from allergen CyPs on B1-B3 sequence of B-cell epitopes. Amino acid sequence (N-H-N) of B1 was replaced by R-G-D of CyPB and amino acid sequence (P-N) of B2 was replaced by K-D of CyPB. Amino acid sequence of $\mathrm{B} 3$ was replaced by whole loop2 (Fig. 6C). That may be the reason that $\mathrm{CyPB}$ is not immunogenic ${ }^{29}$.

In summary, bioinformatics and immunoinformatics analysis illustrated that $\mathrm{CyPE}$ is an essential element of cyclophilin pan-allergen family. $\mathrm{CyPE}$ is low antigenicity but sufficient to induced significant airway inflammation in mouse models and IgE, IgG1, eosinophil infiltration, IL-4 and co-stimulatory molecules of CyPE were markedly lower levels than Der f 29. Structure and immunoinformatics analysis demonstrated 
that $\mathrm{RBM}$ of CyPE may reduce the immunogenicity of $\mathrm{CyPE}$ and structure variation of B-cell epitopes is the reason why CyPB is no IgE activity. This work is of significance in the diagnosis and treatment of allergy.

\section{Author Contributions}

Yuwei Li wrote the main manuscript and performed the experiments presented in Figures 1-4 and analyzed the data. Yuwei Wang performed the experiments presented in Figures 5 and analyzed the data. Bei Jiang and Ruyi Yuan performed the experiments presented in Figures 6 and analyzed the data. Zhigang Liu, Pingchang Yang, Baoqing Sun, and Pixin Ran designed the project, supervised the experiments and wrote the manuscript. All authors reviewed the manuscript.

\section{Funding}

This study was supported by grants from the National Natural Science Foundation of China (No. 81901684, No. 31729002, and No. U1801286), Shenzhen Science and Technology Peacock Team Project (No.KQTD20170331145453160), International Science and Technology Cooperation Project of Shenzhen Science and Technology Plan (No.GJHZ2018041819053), Shenzhen Scientific Technology Basic Research Projects (No.JCYJ20180306171550045), State Key Laboratory of Respiratory Diseases Open Topic: Basic analysis of standardization and clinical diagnosis and treatment of new allergen components in dust mites (No. SKLRD-OP-201909).

\section{Declaration of competing interest}

The authors declare that they have no known competing financial interests or personal relationships that could have appeared to influence the work reported in this paper.

\section{References}

1. Valenta R, ., Steinberger P, ., Duchêne M, ., Kraft D, . Immunological and structural similarities among allergens: prerequisite for a specific and component-based therapy of allergy. Immunology \& Cell Biology. 1996;74(2):187-194.

2. Appenzeller U, Meyer C, Menz G, Blaser K, Crameri R. IgE-mediated reactions to autoantigens in allergic diseases. International archives of allergy and immunology. 1999;118(2-4):193-196.

3. Sabine F, Helmi F, Paul W, Kurt B, Reto C. Cyclophilins, a new family of cross-reactive allergens. European journal of immunology.2015;32(1):10-17.

4. Takahashi N, Hayano T, Suzuki M. Peptidyl-prolyl cis-trans isomerase is the cyclosporin A-binding protein cyclophilin. Nature.1989;337(6206):473.

5. Handschumacher RE, Harding MW, Rice J, Drugge RJ, Speicher DW. Cyclophilin: a specific cytosolic binding protein for cyclosporin A.Science. 1984;226(4674):544-547.

6. Borel JF. Pharmacology of cyclosporine (sandimmune). IV. Pharmacological properties in vivo. Pharmacological reviews.1990;41(3):259-371.

7. Liu J, Farmer Jr JD, Lane WS, Friedman J, Weissman I, Schreiber SL. Calcineurin is a common target of cyclophilin-cyclosporin A and FKBP-FK506 complexes. Cell. 1991;66(4):807-815. 
8. Jain J, McCafffrey PG, Miner Z, et al. The T-cell transcription factor NFATp is a substrate for calcineurin and interacts with Fos and Jun. Nature. 1993;365(6444):352.

9. O'Keefe SJ, Tamura Ji, Kincaid RL, Tocci MJ, O'Neill EA. FK-506-and CsA-sensitive activation of the interleukin-2 promoter by calcineurin. Nature. 1992;357(6380):692.

10. Schiene-Fischer C. Multidomain peptidyl prolyl cis/trans isomerases.Biochimica et Biophysica Acta (BBA)-General Subjects.2015;1850(10):2005-2016.

11. Kieffer L, Thalhammer T, Handschumacher R. Isolation and characterization of a 40-kDa cyclophilinrelated protein. Journal of Biological Chemistry. 1992;267(8):5503-5507.

12. Calderón MA, Bousquet J, Canonica GW, Cardell LO, Kleine-Tebbe J, Demoly P. Guideline recommendations on the use of allergen immunotherapy in house dust mite allergy: time for a change? Journal of Allergy $\&$ Clinical Immunology. 2017;140(1):41.

13. Horner W, Reese G, Lehrer S. Identification of the allergen Psi c 2 from the basidiomycete Psilocybe cubensis as a fungal cyclophilin. International archives of allergy and immunology.1995;107(1-3):298-300.

14. Lindborg M, Magnusson CG, Zargari A, et al. Selective cloning of allergens from the skin colonizing yeast Malassezia furfur by phage surface display technology. Journal of investigative dermatology.1999;113(2):156161.

15. Kumar S, Stecher G, Tamura K. MEGA7: molecular evolutionary genetics analysis version 7.0 for bigger datasets. Molecular biology and evolution. 2016;33(7):1870-1874.

16. Thompson JD, Gibson TJ, Plewniak F, Jeanmougin F, Higgins DG. The CLUSTAL_X windows interface: flexible strategies for multiple sequence alignment aided by quality analysis tools. Nucleic acids research. 1997;25(24):4876-4882.

17. Gasteiger E, Hoogland C, Gattiker A, Wilkins MR, Appel RD, Bairoch A. Protein identification and analysis tools on the ExPASy server. In:The proteomics protocols handbook. Springer; 2005:571-607.

18. Jones DT. Protein secondary structure prediction based on position-specific scoring matrices. Journal of molecular biology.1999;292(2):195-202.

19. Kiefer F, Arnold K, Künzli M, Bordoli L, Schwede T. The SWISS-MODEL Repository and associated resources. Nucleic acids research.2008;37(suppl_1):D387-D392.

20. Bowie JU, Luthy R, Eisenberg D. A method to identify protein sequences that fold into a known threedimensional structure.Science. 1991;253(5016):164-170.

21. Ramachandran S, Kota P, Ding F, Dokholyan NV. Automated minimization of steric clashes in protein structures. Proteins: Structure, Function, and Bioinformatics. 2011;79(1):261-270.

22. Yang H, Chen H, Jin M, Xie H, He S, Wei J-F. Molecular cloning, expression, IgE binding activities and in silico epitope prediction of Per a 9 allergens of the American cockroach. International journal of molecular medicine. 2016;38(6):1795-1805.

23. Ansari HR, Raghava GP. Identification of conformational B-cell Epitopes in an antigen from its primary sequence. Immunome research. 2010;6(1):6.

24. Zheng L-N, Lin H, Pawar R, Li Z-X, Li M-H. Mapping IgE binding epitopes of major shrimp (Penaeus monodon) allergen with immunoinformatics tools. Food and Chemical Toxicology.2011;49(11):2954-2960.

25. Wang Y, Han R, Zhang W, et al. Human CyP33 binds specifically to mRNA and binding stimulates PPIase activity of hCyP33. FEBS letters. 2008;582(5):835-839.

26. Trandinh CC, Pao G, Saier Jr M. Structural and evolutionary relationships among the immunophilins: two ubiquitous families of peptidyl-prolyl cis-trans isomerases. The FASEB journal.1992;6(15):3410-3420. 
27. Sporik R, Holgate ST, Platts-Mills TA, Cogswell JJ. Exposure to house-dust mite allergen (Der $\mathrm{p}$ I) and the development of asthma in childhood: a prospective study. New England journal of medicine.1990;323(8):502-507.

28. Jiang C, Fan X, Li M, Xing P, Liu Z. Characterization of Der f 29, a new allergen from dermatophagoides farinae. American Journal of Translational Research. 2015;7(7):1303-1313.

29. Wang H, Mo L, Xiao X, et al. Pplase of Dermatophagoides farinae promotes ovalbumin-induced airway allergy by modulating the functions of dendritic cells in a mouse model. entific Reports. 2017;7:43322.

30. Sanchez-Trincado JL, Marta G-P, A. RP. Fundamentals and Methods for T- and B-Cell Epitope Prediction. Journal of Immunology Research. 2017:1-14.

31. Halim TY, Hwang YY, Scanlon ST, et al. Group 2 innate lymphoid cells license dendritic cells to potentiate memory T H 2 cell responses. Nature immunology. 2016;17(1):57.

32. Gill MA. The role of dendritic cells in asthma. Journal of allergy and clinical immunology. 2012;129(4):889-901.

33. Gao Y, Nish SA, Jiang R, et al. Control of T helper 2 responses by transcription factor IRF4-dependent dendritic cells. Immunity.2013;39(4):722-732.

34. Tussiwand R, Everts B, Grajales-Reyes GE, et al. Klf4 expression in conventional dendritic cells is required for T helper 2 cell responses.Immunity. 2015;42(5):916-928.

35. Salazar F, Ghaemmaghami A. Allergen recognition by innate immune cells: critical role of dendritic and epithelial cells. Frontiers in immunology. 2013;4:356.

36. Jeon W-Y, Shin I-S, Shin H-K, Lee M-Y. Samsoeum water extract attenuates allergic airway inflammation via modulation of Th1/Th2 cytokines and decrease of iNOS expression in asthmatic mice. BMC complementary and alternative medicine. 2015;15(1):47.

37. Mikol V, Walkinshaw KMD. X-Ray Structure of a Cyclophilin B/Cyclosporin Complex: Comparison with Cyclophilin A and Delineation of its Calcineurin-Binding Domain. Proceedings of the National Academy of Sciences of the United States of America.1994;91(11):5183-5186.

Figure legends

Figure 1. Phylogenetic analysis and three-dimensional structure model of CyPs. (A) Evolutionary tree of CyPs. Allergens CyPs are highlighted by red background and CyPE are highlighted by green background. (B) Three-dimensional structure model of CyPA. (C) Three-dimensional structure model of CyPE. Cartoon representation of a single subunit of $\mathrm{CyPs}$ which shows the overall $\alpha / \beta$ barrel fold. The $\alpha$-helices and $\beta$-strands in CyPs conformation is highlighted in purple and green, respectively.

Figure 2. B-cell epitopes and antigenicity of allergen CyPs (A) B-cell epitopes were labeled in sequence comparison of $\mathrm{CyPE}$ and other CyPs allergens from different species. Sequence comparison of different CyPs performed by multiple alignment. 2D elements were depicted as blue barrels ( $\alpha$-helices) and green arrows ( $\beta$-sheets). Sequences of B-cell epitopes were framed in different colors and named B1-B3. The common epitopes of CyPs were highlighted by red background. Residues conserved among the five species are highlighted by red font and marked "* " under the sequences, while these only conserved in the four first species were marked ". or : " under the sequences. Active site residues were marked "** under the sequences. (B) BcePred's antigenicity plots of the allergen CyPs. B-cell epitopes were framed in red box and corresponded to B1-B3 of sequence comparison. (C) B-cell epitopes B1-B3 of CyPE and other CyPs allergens are labeled the superimposed $\alpha$-carbon backbones structure of CyPs. Superimposition and drawing was performed using Discovery Studio 2.5.

Figure 3. Expression and immunological characterization of CyPs. (A) SDS-PAGE analysis of the purified CyPs. Lane M: Protein Marker. Lane 1: the purified CyPA; Lane 2: the purified CyPB; Lane 3: the purified 
CyPE. (C) The specific IgE reactivity to allergen CyPs by ELISA. $n$, the serum from healthy subjects $(\mathrm{n}=3)$; P1-P14 reordered according to optical density (OD), the serum from the patients with dust-mite allergy.

Figure 4. Airway allergy is induced by different CyPs. (A) Schematic representation of establishment of mouse model. (B) The level of IgE were detected by ELISA. The serum was collected on day 23, and the levels of specific serum IgE were measured. (C) The level of IgG1 were detected by ELISA. The serum was collected on day 23, and the levels of specific serum IgG1 and IgE were measured. (D) CyPs triggers an eosinophilia. representative flow profile of Siglec- $\mathrm{F}^{+}$eosinophils in BALF. (E) Lung pathology of animals challenged with PBS and different CyPs. These data (means \pm SEMs) were generated from one of three experiments $(\mathrm{n}=4-6)$ and processed with GraphPad software. The significant differences between two groups were tested by two-tailed T-test. ${ }^{* *} \mathrm{p}<0.01 .{ }^{* * *} \mathrm{p}<0.001 . \mathrm{ns}$, no significant difference.

Figure 5. CyPE induces Th2-type response in spleen by modulating DCs. (A) The spleen cells were cultured with CyPs for 3 days, then analyzed by FACS. The spleen cells of IL- 4 and IFN- $\gamma$ were counted with GraphPad software. (B) The expression levels of CD83, CD80 and MHC II of DCs were tested by flow cytometry and processed with GraphPad software. DC2.4 cells were seeded into 6-well plates for overnight culture and then stimulated with $\mathrm{CyPE}$ for 24 hours. These data were generated from three independent experiments. The significant differences between two groups were tested by two-tailed T-test. ${ }^{*} \mathrm{p}<0.05$. $*^{* *} \mathrm{p}<0.01 . \mathrm{ns}$, no significant difference.

Figure 6. Structure and Sequence analysis of allergen and no-allergen CyPs. (A) The superimposed structure of allergen CyPs was performed using Discovery Studio 2.5. (B) The structure of no-allergen CyPs. (C) Sequence analysis of allergen and no-allergen CyPs. The common epitopes of CyPs were highlighted by red background, and corresponding residues of no-allergen CyPs are marked by blue box.

Table 1. Features of CyPs

\begin{tabular}{llllllll}
\hline Allergen & Immuno- positive rate & Domain & Number of amino acids & molecular weight & Theoretical pI & GRAVY & Ins \\
\hline CyPE-A & - & CyP-A & 165 & $18.4 \mathrm{kDa}$ & 9.35 & -0.432 & 17. \\
Der f 29 & $35 / 41(85 \%)$ & CyP-A & 164 & $17.7 \mathrm{kDa}$ & 9.17 & -0.209 & 21.2 \\
Asp f 11 & $27 / 30(90 \%)$ & CyP-A & 178 & $19.5 \mathrm{kDa}$ & 8.87 & -0.455 & 23. \\
Asp f 27 & $30 / 40(75 \%)$ & CyP-A & 163 & $17.7 \mathrm{kDa}$ & 7.75 & -0.250 & 11. \\
Mala s 6 & $20 / 97(21 \%)$ & CyP-A & 162 & $17.2 \mathrm{kDa}$ & 8.79 & -0.083 & 18. \\
Rhi o 2 & $74 / 114(65 \%)$ & CyP-A & 164 & $17.8 \mathrm{kDa}$ & 8.38 & -0.321 & 19. \\
Bet v 7 & $(20.8 \%)$ & CyP-A & 173 & $18.3 \mathrm{kDa}$ & 8.68 & -0.234 & 15.2 \\
Cat r 1 & $5 / 15(33 \%)$ & CyP-A & 172 & $18.3 \mathrm{kDa}$ & 8.36 & -0.270 & 21.2 \\
Ole e 15 & $17 / 79(22 \%)$ & CyP-A & 172 & $18.1 \mathrm{kDa}$ & 8.68 & -0.070 & 14. \\
Sola 1 15 & - & CyP-A & 171 & $17.9 \mathrm{kDa}$ & 8.83 & -0.109 & 12.2 \\
CyPB & $0 / 10(0)$ & CyP-B & 224 & $24.8 \mathrm{kDa}$ & 7.66 & -0.308 & 23.5 \\
\hline
\end{tabular}

Table 2. Parameters used for proteins structural assessment

\begin{tabular}{llllll}
\hline protein & Structural assessment methods & Ramachandran plot (\%) & ?-factor & ?-score & ? value \\
\hline CyPE & PROCHECK analysis & $89.8^{\mathrm{E}}$ & $-0.10^{\mathrm{I}}$ & & \\
& & $9.2^{\mathrm{F}}$ & $0.21^{\mathrm{J}}$ & & \\
& & $1.0^{\mathrm{G}}$ & $-0.05^{\mathrm{K}}$ & & \\
& & $0.0^{\mathrm{H}}$ & & & \\
& ProSa & & & & $0.88 \pm 0.05$ \\
& QMEAN & & $-0.22^{\mathrm{I}}$ & & \\
5yzg.1 & PROCHECK analysis & $88.2^{\mathrm{E}}$ & $0.62^{\mathrm{J}}$ & & \\
& & $11.3^{\mathrm{F}}$ & $0.5^{\mathrm{G}}$ & $0.16^{\mathrm{K}}$ & \\
& & & &
\end{tabular}




\begin{tabular}{llllll}
\hline protein & Structural assessment methods & Ramachandran plot (\%) & ?-factor & ?-score & ? value \\
\hline & $0.0^{\mathrm{H}}$ & & & \\
& ProSa & & -8.08 & \\
QMEAN & & & $0.79 \pm 0.05$ \\
\hline
\end{tabular}

${ }^{\mathrm{E}}$ Residues in favorable regions; ${ }^{\mathrm{F}}$ residues in allowed regions; ${ }^{\mathrm{G}}$ residues in generally allowed regions; ${ }^{\mathrm{H}}$ residues in disallowed regions;

I?-factor score of the dihedral bonds; ${ }^{\mathrm{J}}$ ?-factor score of the covalent bonds; ${ }^{\mathrm{K}}$ overall ?-factor score

Table 3. The results of $\mathrm{B}$ cell epitopes predictions of $\mathrm{CyPs}$

\begin{tabular}{|c|c|c|}
\hline & Tools & Location of the prediction results \\
\hline \multirow[t]{4}{*}{$\begin{array}{l}\text { B cell epitope prediction } \\
\text { CyP-ABH domain }\end{array}$} & DNAStar protean & $\begin{array}{l}27-38,67-82,107-111 \\
126-128,152-161\end{array}$ \\
\hline & Cbtope & $67-80,103-115,125-128,134-165$ \\
\hline & BepiPred & $\begin{array}{l}29-33,66-85,95-109,119-122 \\
144-155 .\end{array}$ \\
\hline & Bcepred & $\begin{array}{l}25-34,67-84,99-109,103-115 \\
125-128,139-153\end{array}$ \\
\hline \multirow[t]{4}{*}{ B cell epitope prediction Der f 29} & DNAStar protean & $\begin{array}{l}10-17,22-37,40-52,62-88 \\
103-111,121-125,137-157\end{array}$ \\
\hline & Cbtope & $\begin{array}{l}27-43,67-82,107-111 \\
123-129,142-163\end{array}$ \\
\hline & BepiPred & $\begin{array}{l}12-17,28-33,45-48, \\
66-84,89-93,102-111,143-157 .\end{array}$ \\
\hline & Bcepred & $\begin{array}{l}23-34,39-51,63-77,102-109, \\
126-132,139-162 .\end{array}$ \\
\hline \multirow[t]{4}{*}{ B cell epitope prediction Mala s 6} & DNAStar protean & $\begin{array}{l}8-15,20-32,38-45,60-77,80-95 \\
101-109,120-124,140-161\end{array}$ \\
\hline & Cbtope & $\begin{array}{l}27-39,65-72,91-97,105-115 \\
145-162 .\end{array}$ \\
\hline & BepiPred & $\begin{array}{l}10-15,27-39,42-46,64-76,91-115 \\
141-152\end{array}$ \\
\hline & Bcepred & $\begin{array}{l}24-42,59-74,87-97,100-115 \\
140-151 .\end{array}$ \\
\hline \multirow[t]{4}{*}{ B cell epitope prediction Asp f 27} & DNAStar protean & $\begin{array}{l}10-18,27-51,62-74,77-96 \\
101-110,130-157 .\end{array}$ \\
\hline & Cbtope & $\begin{array}{l}30-43,61-78,88-94,106-111, \\
145-151 .\end{array}$ \\
\hline & BepiPred & $\begin{array}{l}\text { 11-18, 36-48, 65-94, 102-111, } \\
143-154 .\end{array}$ \\
\hline & Bcepred & $\begin{array}{l}36-50,61-76,88-94,100-107 \\
142-152 .\end{array}$ \\
\hline
\end{tabular}

Table 4. Predicted B-cell epitope sequences of allergen CyPs

\begin{tabular}{lll}
\hline allergen & Position & Sequence \\
\hline $\mathrm{CyP}-\mathrm{ABH}$ domain & - & -
\end{tabular}




\begin{tabular}{lll}
\hline allergen & Position & Sequence \\
\hline & $67-78$ & FTNHNGTGGKSI \\
& $103-115$ & SGPNTNGSQFFIT \\
& $144-155$ & ECGSKSGKPNKR \\
Mal s 6 & $36-43$ & RALCTGEK \\
& $62-76$ & QGGDFTKGNGTGGKS \\
& $88-94$ & QLKHDKP \\
Der f 29 & $145-151$ & GSASGTP \\
& $27-43$ & DVVPKTAENFRCLCTG \\
& $67-77$ & FTNHNGTGGKS \\
Asp f 27 & $107-111$ & TNGSQ \\
& $143-157$ & ESYGSQSGKPSKKVTI \\
& $27-39$ & DVVPKTAENFRCLCTG \\
& $65-72$ & FTAGNGTG \\
& $91-97$ & NKPGLLS \\
& $145-162$ & SGSGKPRSRIEIAKCGVC \\
\hline
\end{tabular}
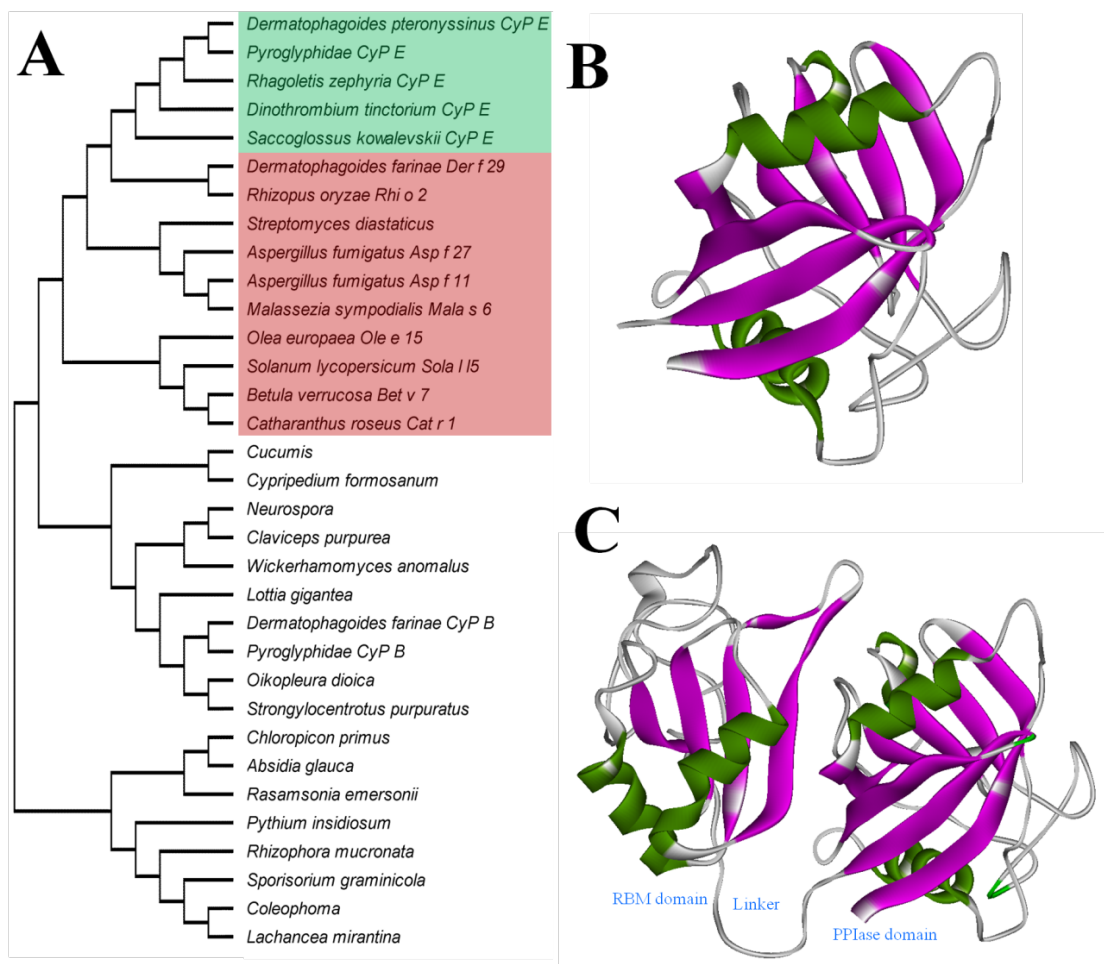

Figure 1 


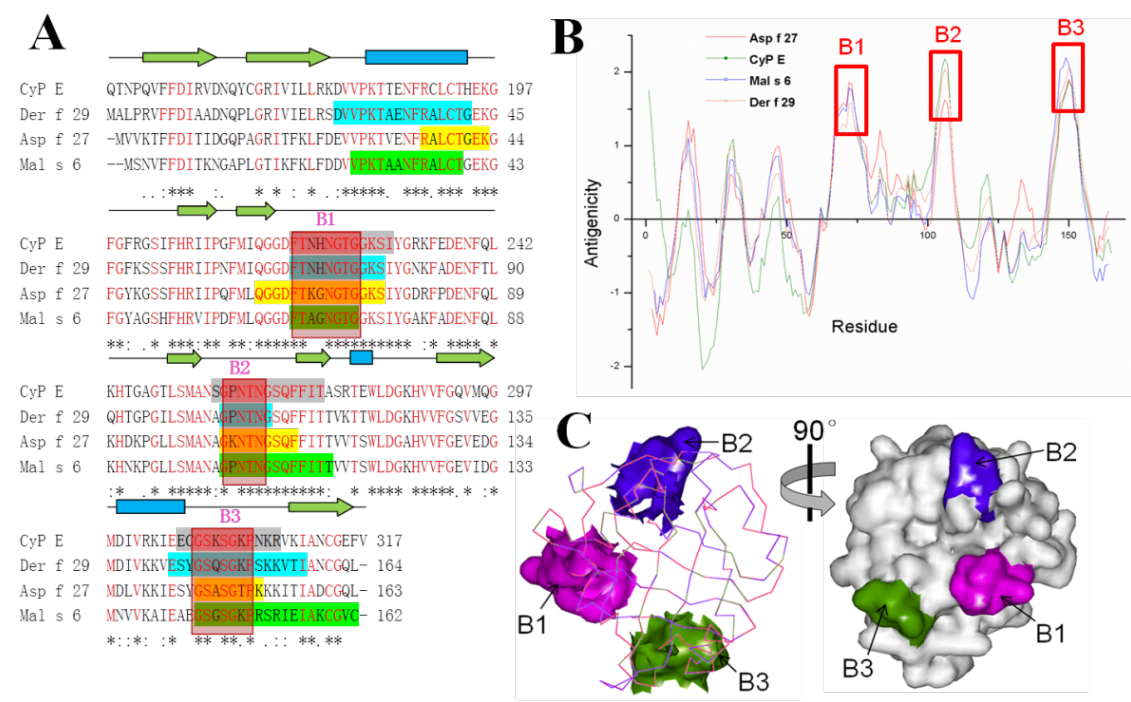

Figure 2
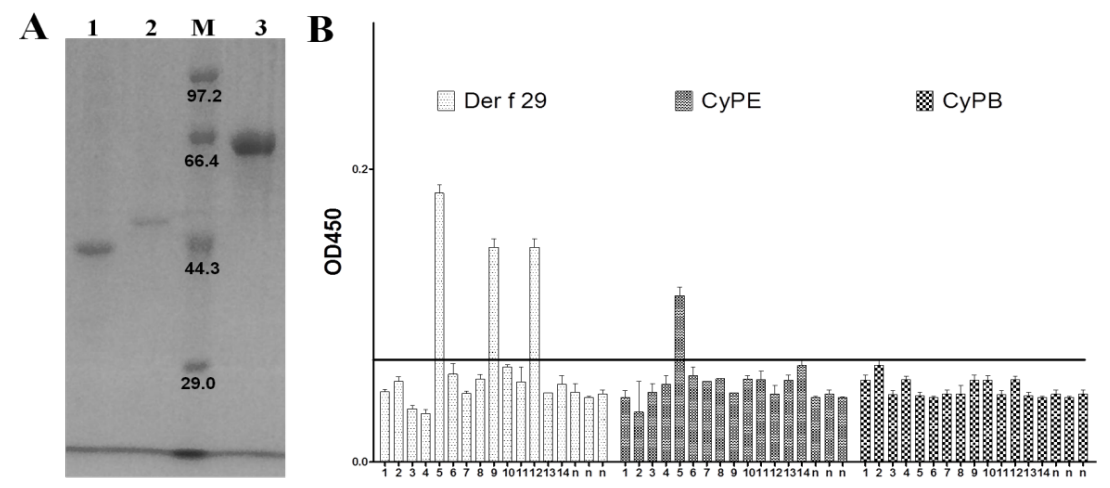

Figure 3 
A

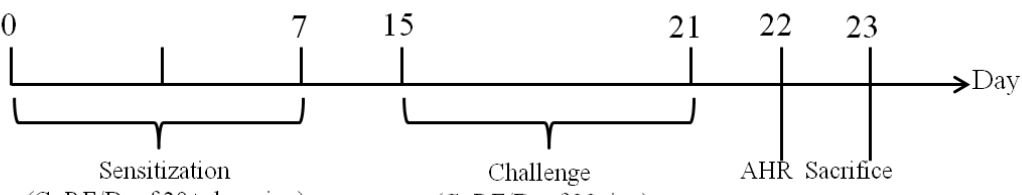
(CyP E/Der f 29+alum, i.p.) (CyP E/Der f 29, i.n.)

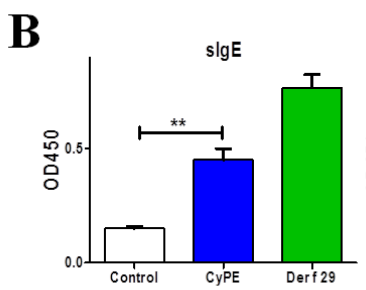

C

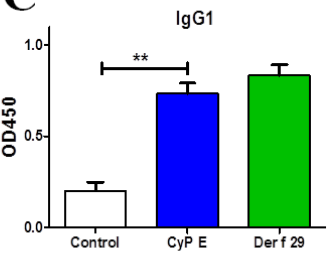

D

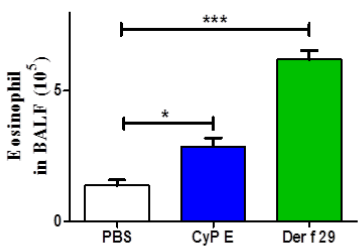

$\mathbf{E}$

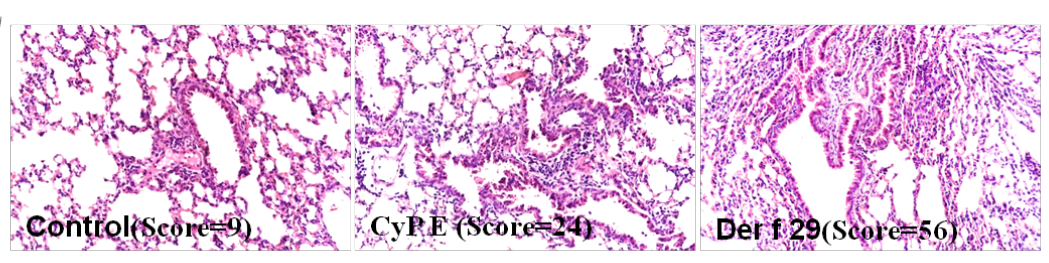

Figure 4
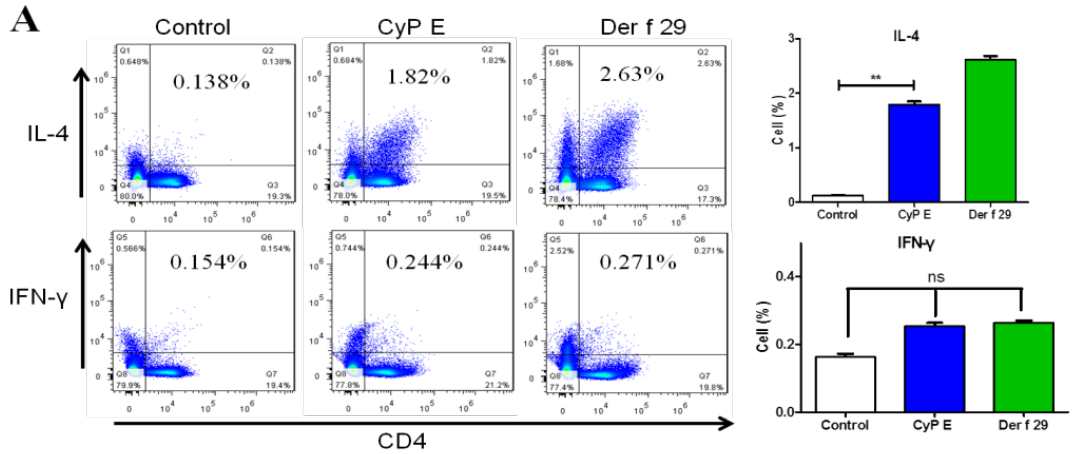

B

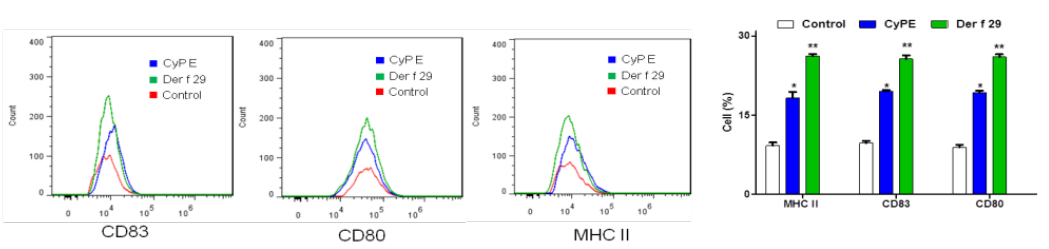

Figure 5 


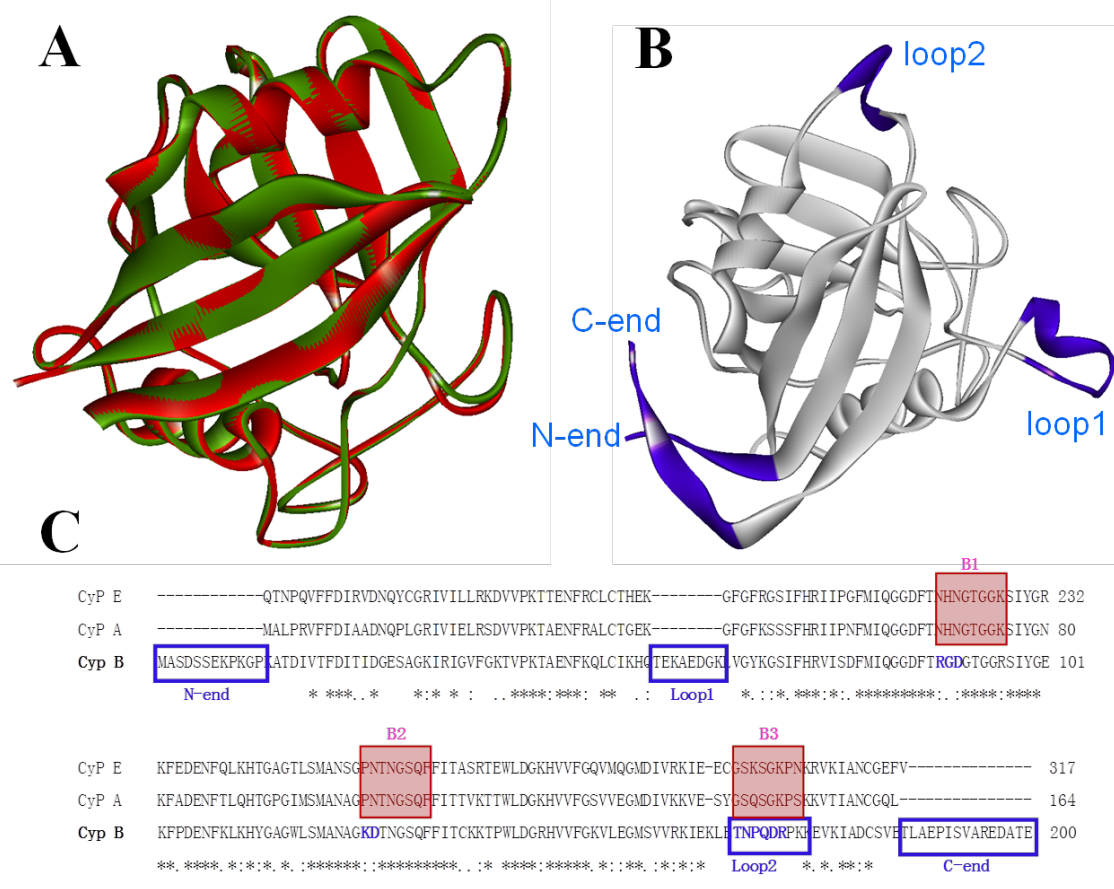

Figure 6 